\title{
Exceptional Dehn filling
}

\author{
Steven Boyer (Université du Québec à Montréal), \\ Cameron McA. Gordon (University of Texas at Austin)
}

October 25 - November 1, 2009

\section{Overview of the Field}

This Research in Team workshop focused on several problems in the theory of exceptional Dehn fillings in 3dimensional topology. Dehn filling is the construction in which you take a 3-manifold $M$, with a distinguished torus boundary component $T$, and glue a solid torus $V$ to $M$ via some homeomorphism from $\partial V$ to $T$. The resulting manifold depends only on the isotopy class (slope) $\alpha$ on $T$ that is identified with the boundary of a meridian disk of $V$, so we denote it by $M(\alpha)$. The construction goes back to Dehn in 1910, who introduced it in the special case where $M$ is the exterior of a knot in $S^{3}$. The Lickorish-Wallace theorem of the early 1960's showed that any closed, connected, orientable 3-manifold can be obtained by Dehn filling the boundary tori of the exterior of some link in $S^{3}$. Consequently, many of the basic problems in 3-manifold topology can been analysed in terms of the operation.

Renewed interest in the construction arose with the ground-breaking work of Thurston in the 1970's, who used it to study hyperbolic geometric structures on 3-manifolds. In particular, Thurston showed that if $M$ is hyperbolic then $M(\alpha)$ is also hyperbolic for all but finitely many slopes $\alpha$ on $T$. When $M$ is hyperbolic but $M(\alpha)$ is not, one says that $(M ; \alpha)$ is exceptional. Although it is clear that one cannot hope to classify all exceptional $(M ; \alpha)$ 's, it turns out that it is relatively rare for a hyperbolic 3-manifold to have two distinct exceptional slopes $\alpha$ and $\beta$ on $T$, and it is not too unreasonable to try to classify all such $(M ; \alpha, \beta)$ 's. This has usually been approached by considering the various different ways in which $M(\alpha)$ and $M(\beta)$ can fail to be hyperbolic, and there has been a lot of progress along these lines. The cases about which least is known is when the boundary of $M$ is a torus and one of the fillings, say $M(\beta)$, is a small Seifert fiber space. This was the focus of the workshop

\section{Recent Developments and Open Problems}

As stated in the Overview, the focus of the workshop was the classification of triples $(M ; \alpha, \beta)$ where $M$ is a hyperbolic 3-manifold with torus boundary and $M(\alpha)$ and $M(\beta)$ are distinct non-hyperbolic Dehn fillings on $M$. There has been considerable progress on this classification over the last 30 years or so, but some problems remain. Let $\mathcal{E}(M)=\{\alpha \mid M(\alpha)$ is not hyperbolic $\}$ be the set of exceptional slopes on the boundary of $M$. Two problems which have been the focus of intense research are:

(A) Understand the structure of $\mathcal{E}(M)$.

(B) Describe the topology of $M$ when $|\mathcal{E}(M)| \geq 2$. 
See the survey [6] for instance.

An essential quantity used to describe results on these problems is the notion of the distance $\Delta(\alpha, \beta)$ (minimal geometric intersection number) between two exceptional slopes $\alpha$ and $\beta$. Two results which exemplify what can occur when the situation described in problem (B) arises are Gordon's theorem: if two toroidal filling slopes are of mutual distance at least 6 , then $M$ is one of four specific manifolds $M_{1}, M_{2}, M_{3}, M_{4}$ [7], and Ni's recent theorem: if $M$ is the exterior of a knot in the 3-sphere which has a non-meridional slope whose associated filling yields a lens space, then $M$ fibres over the circle [11]. One of the key conjectures concerning problem (A) is the following:

Conjecture [C.McA. Gordon] $\# \mathcal{E}(M) \leq 10$ and $\Delta(\mathcal{E}(M)) \leq 8$. Moreover, if $M \neq M_{1}, M_{2}, M_{3}, M_{4}$, then $\# \mathcal{E}(M) \leq 7$ and $\Delta(\mathcal{E}(M)) \leq 5$.

It is shown in [4] that the conjecture holds if the first Betti number of $M$ is at least 2. (Note that it is at least 1.) Lackenby and Meyerhoff have recently announced a proof that the first statement of the conjecture holds in general [8]. Agol has shown that there are only finitely many hyperbolic knot manifolds $M$ with $\Delta(\mathcal{E}(M))>5$ [1], though there is no practical fashion to determine this finite set.

It is a consequence of the Geometrization Conjecture, recently proved by Perelman (c.f. $[9,10])$, that if a 3-manifold is not hyperbolic, then it is either

(1) reducible (contains an essential sphere), or

(2) toroidal (contains an essential torus), or

(3) a Seifert fiber space over $S^{2}$ with at most 3 exceptional fibers.

Moreover, a manifold of type (3) is either

(a) $S^{3}$, or

(b) a lens space, or

(c) a small Seifert fiber space (SSFS), i.e. one with base $S^{2}$ and exactly 3 exceptional fibers.

Finally, case 3(c) splits into two subcases (i) finite fundamental group, and (ii) infinite fundamental group.

The problems have usually been approached by considering the various possibilities (1), (2), (3)(a), (3)(b), (3)(c)(i) or 3(c)(ii), for the pair of non-hyperbolic manifolds $M(\alpha)$ and $M(\beta)$. Perhaps surprisingly, the least tractable cases are when $M(\alpha)$ or $M(\beta)$ (or both) is a SSFS. When neither is a SSFS, the best possible upper bounds for $\Delta(\alpha, \beta)$ are essentially known, as a result of the work of several people (there are only two cases left). Further, the conjecture above has been verified in all cases. When $M(\beta)$ is a SSFS and $M(\alpha)$ belongs to one of the classes of non-hyperbolic manifolds listed, an important problem is to obtain the optimal upper bound on $\Delta(\alpha, \beta)$.

The case where $M(\alpha)$ is reducible was considered in [2] and [3]. Building on [3], but introducing new tangle-theoretic techniques, the optimal bound $\Delta(\alpha, \beta)=1$ in the case where $M(\beta)$ has finite fundamental group was established in [5]. Suppose now that $M(\alpha)$ is toroidal. In this case there is an $m$-punctured essential genus 1 surface $F$ of slope $\alpha$ properly embedded in $M$. Assume that $F$ is chosen to minimize $m$ among all such surfaces. It is expected that if $\Delta(\alpha, \beta)>5$, then $M$ is the exterior of the figure eight knot and $F$ is separating with $m=2$.

\section{Scientific Progress Made}

The precise focus of this Research in Team workshop was the case where $M(\alpha)$ is toroidal and $M(\beta)$ is a SSFS. During our week at BIRS, we essentially completed the proof that $\Delta(\alpha, \beta) \leq 5$ if $F$ does not split $M$ into $I$-bundles and $m \geq 3$. This was achieved by enhancing the method used in $[2,3]$ based on the JSJ decomposition. A paper Characteristic Submanifold Theory and Toroidal Dehn Filling I by S. Boyer, C. McA. Gordon, and X. Zhang is currently being prepared which details this advance. We also made significant advances on the case $m \leq 2$ where a combination of the JSJ technique and tangle-theoretic technique introduced in [5] is used. This work will appear in a second paper Characteristic Submanifold Theory and Toroidal Dehn Filling II that we are currently working on. 


\section{References}

[1] I. Agol, Bounds on exceptional Dehn filling II, preprint (2008), arXiv:0803.3088.

[2] S. Boyer, M. Culler, P. Shalen, and X. Zhang, Characteristic subsurfaces and Dehn fillings, Trans. Amer. Math. Soc. 357 (2005), 2389-2444.

[3] —-, Characteristic subvarieties, character varieties, and Dehn fillings, Geom. \& Top. 12 (2008), 233-297.

[4] S. Boyer, C. McA. Gordon and X. Zhang, Dehn fillings of large hyperbolic 3-manifolds, J. Diff. Geom. 58 (2001), 263-308.

[5] —-, Reducible and finite Dehn fillings, J. Lond. Math. Soc., to appear.

[6] C. McA. Gordon, Dehn filling: a survey, in: Knot Theory, Banach Center Publications 42 (1998) 129144.

[7] —-, Boundary slopes of punctured tori in 3-manifolds, Trans. Amer. Math. Soc. 350 (1998) 17131790.

[8] M. Lackenby and R. Meyerhoff, The maximal number of exceptional Dehn surgeries, preprint (2008), arXiv:0808.1176.

[9] J. Morgan and G. Tian, Ricci flow and the Poincaré Conjecture, Clay Mathematics Monographs 3, American Math. Society (2007).

[10] - Completion of the proof of the Geometrization Conjecture, preprint (2008), arXiv:math.DG/0809.4040v1.

[11] Y. Ni, Knot Floer homology detects bred knots, Invent. Math. 170 (2007), 577-608. 